\title{
How might Covid-19 affect the biology curricula of the future? Two principles for curriculum developers to consider
}

\author{
Judith M. D. Roberts ${ }^{1}$
}

Accepted: 9 December 2020 / Published online: 5 January 2021

(C) UNESCO IBE 2021

\begin{abstract}
Understanding the lifestyle changes that authorities have requested or required in response to Covid-19 requires some biological knowledge. Therefore, articulations of intended biology learning at the school level will need to be evaluated, to see if they continue to be fit for purpose in light of the pandemic. This article proposes two principles of curriculum development and applies them to the (re)development of biology curricula in response to Covid-19. Firstly, while Covid-19 provides a vivid contextualisation of many biological concepts, it does not change the underlying concepts themselves. Moreover, it will not take long before it passes from being contemporary experience to a historical case study. Care is, therefore, needed to retain focus on the core concepts of biology, rather than allocating too much time to the particulars of the Covid-19 case. Secondly, biology curricula are often used to educate a population about public health. However, policymakers should be aware that knowledge alone is often insufficient to generate healthy behaviours.
\end{abstract}

Keywords Covid-19 - Biology curriculum · Curriculum design · Curriculum principles · Powerful knowledge $\cdot$ Public health

The Covid-19 pandemic is a major event that will influence many aspects of life, including education, for decades to come. In my work as a biology specialist on Cambridge International's Curriculum Programmes team, I have helped develop and evaluate biology curricula for many contexts, including several national curricula. Here I focus on "intended curricula", i.e., articulations of the expectations for the biology that children should learn, as specified by a Ministry of Education or other body (terminology from the TIMSS curriculum model, e.g., Mullis and Martin 2013).

Judith M. D. Roberts

judith.roberts@cambridgeinternational.org

1 The Triangle Building, Cambridge Assessment International Education, Shaftesbury Road,

Cambridge CB2 8EA, UK 
For each biology curriculum I have been involved in, the curriculum development teams have selected public health messages and contemporary examples that are important to their contexts. As Covid-19 is a global pandemic of society-changing severity, future curriculum developers are likely to reference it specifically. If done well, this approach will provide a vivid and contemporary contextualisation of many biological principles. If done unthinkingly, however, it could result in unbalanced curricula that focus on Covid-19 to the detriment of other important areas of biology, such as ecology, plant sciences and noncommunicable diseases. These intended curricula also risk becoming quickly outdated as Covid-19 passes from being current experience to a historical case study. This article urges caution to developers of the next iteration of intended biology curricula based on two principles of curriculum design.

\section{Principle 1: Curricula should focus on the most important aspects of each subject and be changed as infrequently as possible}

Curriculum design is never simple. If there is too much specified content, there will be insufficient time to teach it well and contextualise it for each class of learners. Too little content, by contrast, increases the likelihood of missing important knowledge, understanding, and skills, thereby eroding curriculum entitlement (Oates 2011). Poorly articulated progression pathways can lead to repetition or gaps rather than an intended spiral (Bruner 1960). In addition, regular curriculum changes made to keep things "current" and "relevant" require resources to be updated, so teachers can no longer reuse tried and tested lessons. Hence, there is both an opportunity cost and a financial cost associated with curriculum revisions (Oates 2011).

Michael Young, from University College London, proposed that curricula should be designed based around "powerful knowledge". These are the key ideas that take learners beyond the limits of their own experiences, give them access to more reliable explanations of the world, and provide a language for engaging in intellectual debates (Young 2008). Although the current pandemic is an extreme case, it exemplifies well-established biological "powerful knowledge" relating to human health, such as:

- the germ theory of disease (that disease can be caused by microscopic organisms, including viruses, that infect hosts)

- adaptive immunity requires an individual to have prior exposure to a pathogen or vaccine

- herd immunity can prevent outbreaks if sufficient members of a population have (adaptive) immunity

- in cases where pathogens pass to new hosts (as seems likely in this case) there is no pre-existing immunity, so the pathogen can spread rapidly

- the transmission potential of a disease can be measured, modelled, and decreased through actions such as handwashing, disinfecting surfaces, and social distancing

- habitat changes can influence the probability of zoonotic infections by changing the frequency and types of interactions between humans and other species.

If these and similar statements are already present in biology curricula, then Covid-19 does not need to be included as a new "concept", but instead could be referenced as a case study or context for understanding and applying this powerful knowledge. This could be done 
by reviewing approaches used to implement the curriculum (for example in schemes of work and textbooks) and assessment questions, without having to reissue the underlying intended curriculum.

A convenient way to review many science curricula simultaneously is to look at the content assessed by TIMSS for learners in Grade 4 and Grade 8. The TIMSS methodology features extensive consultation and review by science education specialists in each of the participating countries. This work results in an assessment framework that emphasises consensus and describes educational goals considered important by a significant number of these countries (Martin and Mullis 2006).

The TIMSS 2019 assessment framework includes "Human Health" as one of the topic areas for life sciences for Grade 4 and Grade 8 participants (Mullis et al. 2016). Specifically, at Grade 4 learners are expected to understand the transmission, prevention, and symptoms of communicable diseases by being able to:

(a) Relate the transmission of common communicable diseases to human contact (e.g., touching, sneezing, coughing).

(b) Identify or describe some methods of preventing disease transmission (e.g., vaccination, washing hands, avoiding people who are sick); recognize common signs of illness (e.g., high body temperature, coughing, stomach ache).

Grade 8 learners are also expected to understand something of the causes and immune response to disease, as well as disease transmission and prevention:

(a) Describe causes, transmission, and prevention of common diseases (e.g., influenza, measles, malaria, HIV).

(b) Describe the role of the body's immune system in resisting disease and promoting healing (i.e., antibodies in the blood help the body resist infection and white blood cells fight infection).

Although providers of intended curricula will need to review their own curricula in terms of the concepts most relevant to Covid-19, the consensus statements in the TIMSS assessment framework suggest that many curricula will already include most, if not all, of the relevant concepts related to human health (perhaps excepting zoonosis). If any key concepts are omitted, then this should be addressed by developers. However, it is likely that many curricula will require only relatively minor tweaks for the next iteration (e.g., adding Covid-19 as a specified example if desired), rather than major rewrites. By limiting the changes in the underlying intended curriculum, more attention can be given to refining the curriculum implementation.

Curriculum implementation is ultimately the responsibility of individual teachers, and there are many ways they may engage their learners with biological concepts and ask them to apply their learning. For example, once learners have understood the concept of herd immunity (which is not controversial when applied to communicable diseases in general), they could discuss the highly controversial ethical questions around Covid-19 control approaches based on herd immunity. Similarly, once learners know the principles of vaccine development, they could consider the biological and ethical questions regarding the use of challenge trials (both historically for smallpox and for Covid-19). When considering human impacts on the environment, learners could use Covid-19 as a case study to consider the conditions that promote zoonotic infections. They might also research the 
environmental effect of the considerable global slowing of modern human activities (for which Rutz et al. (2020) coined the term "anthropause").

\section{Principle 2: Biology curricula can educate about public health messages, but knowledge alone does not guarantee behavioural change}

National (or equivalent) school curricula are attractive places to include public health messages, as they ensure dissemination of locally relevant messages to every child irrespective of geography or socio-economic group (Böttiger and Van Aken 2015). However, impact studies show that knowledge about a public health issue does not automatically translate into healthier behaviours.

A wide range of public health messages may be considered relevant to a curriculum, such as content on diet, reproductive and maternal health, smoking, and first aid, as well as the avoidance of particular infections such as HIV. Jepson et al. (2010) conducted a metareview with the aim of evaluating the effectiveness of several public health interventions. They commented that "although many of the interventions currently include educational components, few explicitly attempt to evaluate their success" (Jepson et al. 2010).

Where outcomes have been measured, curriculum-based approaches have been found to have some effect in increasing physical activity during the school day (especially during breaks and lunch times), and in promoting the consumption of fruit and vegetables (Jepson et al. 2010). However, information alone was found to be insufficient to change behaviours in interventions aiming to decrease or prevent smoking and illegal drug use in young people. In their study of drug use, Faggiano and colleagues (2014) found that the more successful interventions combined knowledge with training in both social competence skills (to combat issues such as low self-esteem and difficulties in coping with anxiety) and social norms (to demonstrate that illegal drug use is not normative).

Curriculum developers should not assume that knowledge of public health messages is sufficient to promote healthy behaviour. That said, it is reasonable to assume that knowledge is a necessary prerequisite for making healthy choices. Data emerging from the Covid-19 pandemic suggest that health inequalities, together with other socio-economic factors, lead to different morbidity and mortality rates in different groups (e.g., Bambra et al. 2020). Therefore, national biology curriculum designers may wish to work with local public health experts to identify if there are particular issues (e.g., common chronic diseases) that might usefully be addressed in future biology curricula. However, although school curricula are good vehicles to communicate knowledge that promotes healthy patterns for life, they are not able to respond quickly enough to rapidly emerging public health issues.

In the case of Covid-19, public health messages have been disseminated through mass media, not through school curricula, and it is likely that a similar approach would be taken for any future pandemics of similar or greater severity. However, alongside authentic health messages there has also been the dissemination of false messages, misinformation, and hoaxes (reported, for example, by Cellan-Jones 2020). Developers of future biology curricula should also consider how to give their learners the knowledge and skills they need to evaluate health information in addition to particular skills such as effective handwashing.

To conclude, school biology curricula cannot be the only means by which public health is attained and maintained. If attempts are made to redevelop intended curricula so that all future learners understand the particulars of Covid-19, then other areas of biology would 
receive insufficient attention. By keeping a focus on "powerful knowledge" to determine the core concepts in a curriculum, developers can avoid this imbalance while allowing teachers to flexibly implement the curriculum as they wish, selecting when and how to use Covid-19 as an example or case study. However, developers of intended national curricula for biology also have a responsibility to ensure that all children in their areas, regardless of socio-economic group or geography, have sufficient knowledge and understanding to make informed lifestyle choices and respond to future health crises. This will include being able to critically evaluate health messages from different sources.

\section{References}

Bambra, C., Riordan, R., Ford, J., \& Matthews, F. (2020). The Covid-19 pandemic and health inequalities. Journal of Epidemiology and Community Health, 74, 964-968. https://doi.org/10.1136/jech-2020214401.

Böttiger, B. W., \& Van Aken, H. (2015). Kids save lives. Resuscitation, 94, A5-A7. https://doi. org/10.1016/j.resuscitation.2015.07.005.

Bruner, J. S. (1960). The process of education (2nd revised edition). Cambridge, MA: Harvard University Press.

Cellan-Jones, R. (2020, February 26). Coronavirus: Fake news is spreading fast. BBC News. https://www. bbc.com/news/technology-51646309.

Faggiano, F., Minozzi, S., Versino, E., \& Buscemi, D. (2014). Universal school-based prevention for illicit drug use. Cochrane Database of Systematic Reviews, 12, 1-142. https://doi.org/10.1002/14651858. CD003020.pub3.

Jepson, R. G., Harris, F. M., Platt, S., \& Tannahill, C. (2010). The effectiveness of interventions to change six health behaviours: A review of reviews. BMC Public Health, 10(1), 538. https://doi. org/10.1186/1471-2458-10-538.

Martin, M. O., \& Mullis, I. V. S. (2006). TIMSS purpose and design. In S. J. Howie \& T. Plomp (Eds.), Contexts of learning mathematics and science: Lessons learned from TIMSS. London: Routledge.

Mullis, I. V. S., \& Martin, M. O. (Eds.) (2013). TIMSS 2015 assessment frameworks. Chestnut Hill, MA: TIMSS \& PIRLS International Study Center, Boston College.

Mullis, I. V. S., Martin, M. O., Goh, S., \& Cotter, K. (2016). TIMSS 2015 encyclopedia - TIMSS 2015. Chestnut Hill, MA: TIMSS \& PIRLS International Study Center, Boston College. http://timssandpirls. bc.edu/timss2015/encyclopedia/.

Oates, T. (2011). Could do better: Using international comparisons to refine the National Curriculum in England. The Curriculum Journal, 22(2), 121-150. https://doi.org/10.1080/09585176.2011.578908.

Rutz, C., Loretto, M., Bates, A. E., Davidson, S. C., Duarte, C. M., Jetz, W., et al. (2020). Covid-19 lockdown allows researchers to quantify the effects of human activity on wildlife. Nature Ecology \& Evolution, 4, 1156-1159. https://doi.org/10.1038/s41559-020-1237-z.

Young, M. (2008). From constructivism to realism in the sociology of the curriculum. Review of Research in Education, 32, 1-28.

Publisher's Note Springer Nature remains neutral with regard to jurisdictional claims in published maps and institutional affiliations.

Judith Roberts trained at the University of Cambridge, starting as a biologist and specialising in parasite immunology for her $\mathrm{PhD}$. During her time as a researcher, she realised that she was much more interested in teaching and learning than laboratory research. She qualified as a school teacher in 2010, received a Masters of Education in 2012, and joined Cambridge International in 2013. Since then she has worked on intended curricula for 3 to 18 year olds, and the associated materials for curriculum implementation, for a wide variety of countries. 